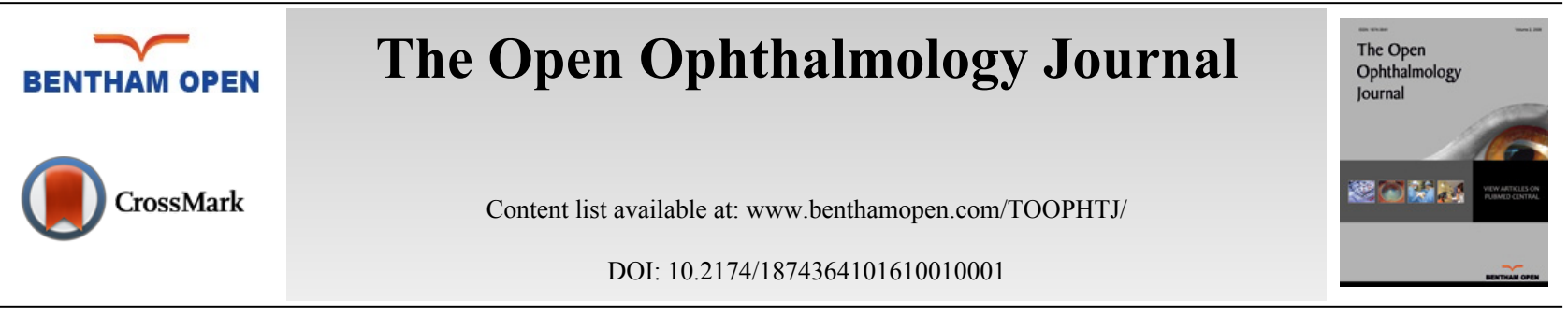

\title{
Morphologic Changes in Patient with Drusen and Drusenoid Pigment Epithelial Detachment after Intravitreal Ranibizumab for Choroidal Neovascular Membrane : A Case Report
}

\author{
Sukjin Kim, Jeongjae Oh and Kiseok Kim ${ }^{*}$ \\ Department of Ophthalmology, Saevit Eye Hospital, Goyangsi, Korea
}

\begin{abstract}
The authors present a case of morphologic changes of drusen and drusenoid pigment epithelial detachment (DPED) after treating choroidal neovascularization (CNV) using ranibizumab in age-related macular degeneration (AMD). A 71-year-old woman has noticed mild visual acuity deterioration in the right eye for several months. She was presented with some drusen and DPED associated with CNV. This patient was given intravitreal injection of $0.5 \mathrm{mg}$ of ranibizumab five times at monthly intervals for treating CNV. DPED in the temporal and drusen in the superior to macula were diminished, which continued up to 2 months. Intravitreal ranibizumab injection may have influenced with diminishment of drusen and DPED. After 2 months, CNV was recurred.
\end{abstract}

Keywords: Age-related macular degeneration, anti-VEGF (vascular endothelial growth factor), drusen, drusenoid pigment epithelial detachment, ranibizumab.

\section{INTRODUCTION}

Drusenoid pigment epithelial detachment (DPED) is defined as a fairly well-circumscribed, shallow elevation of the retinal pigment epithelium, formed by a confluence of soft drusen, often located in the central macula [1]. Disappearance of drusen or DPED has been shown after laser photocoagulation, verteporfin photodynamic therapy, haemorheopheresis and vitrectomy [2 - 9]. Ranibizumab (Lucentis, Genentech, South San Francisco, CA) as a treatment for exudative AMD inhibits a number of subtypes of vascular endothelial growth factor (VEGF). VEGF may trigger the growth of CNV, resulting in the exudative AMD [8]. However, the relationship between VEGF and drusen or DPED was not known [10 - 12]. We report diminishment of drusen and DPED after intravitreal ranibizumab injection for treating $\mathrm{CNV}$, but $\mathrm{CNV}$ was recurred.

\section{CASE REPORT}

A 71-year-old woman has noticed mild visual acuity deterioration in the right eye for several months. Best corrected visual acuity (BCVA) in the right eye was 20/40. Right eye biomicroscopy revealed centrally located DPED with diffuse coalesced soft drusen and retinal hemorrhage at the macular and paramacular region. Hyperfluorescence of fluorescein angiography (FFA) and hypo-reflectivity of OCT (Stratus OCT; OCT3; Carl Zeiss Meditec, Jena, Germany) correspond to CNV with DPED (Figs. 1A, 1C, 1E, 2A and 2E). DPED area was identified by FFA. She underwent intravitreal injections of $0.5 \mathrm{mg}$ ranibizumab five times over 6 months for treating CNV. After 5 intravitreal injections on a monthly basis, DPED located temporal to macula is diminished. Drusen at superotemporal is diminished over time as well (Figs. 1B, 1F, 2B and 2F). CNV was regressed (Fig. 1D) and BCVA of 20/40 improved to 20/25 after 6 months from the initial examination. Reduction of drusen and DPED has continued up to 2 months after initial diminishment (Figs. 2C, 2G). After 2 months, CNV was recurred (Figs. 2D, 2H).

\footnotetext{
* Address correspondence to this author at the Department of Ophthalmology, Saevit Eye Hospital \#1334-3 Baekseok-dong, Ilsan dong-gu, Goyangsi, Gyeonggi-do, 410-817, Korea; Tel: 82-31-900-7700; Fax: 82-31-900-7777; E-mail: kiseok70@gmail.com
} 


\section{DISCUSSION}

Regression of drusen or DPED following intravitreal anti-VEGF therapy was uncommon. Some reports about the regression of drusen and DPED after anti-VEGF therapy are in the literature [5, 6]. Krishnan et al. [5] described 3 eyes of 2 patients who demonstrated a regression of drusen and DPED after anti-VEGF therapy. One patient was DPED with occult CNV at both eyes, and decreased drusen and DPED after 8 months with 3 intravitreal bevacizumab injections in the right eye and 2 intravitreal bevacizumab injections in the left eye. The other patient has left soft drusen and DPED with classic CNV, 6 months after the initial treatment, the number and size of drusen appeared reduced with 5 left intravitreal ranibizumab injections. One prospective pilot study showed that intravitreal ranibizumab demonstrated anatomic and functional benefit in patients with symptomatic DPED without CNV in AMD. That study was prospective, interventional pilot study and includes six eyes in six patients [6]. All 9 cases, no eyes developed CNV or GA, and all eyes improved in visual acuity. However, the use of intravitreal pegaptanib has been reported in DPED, resulting in secondary atrophy with poor visual outcome [7].

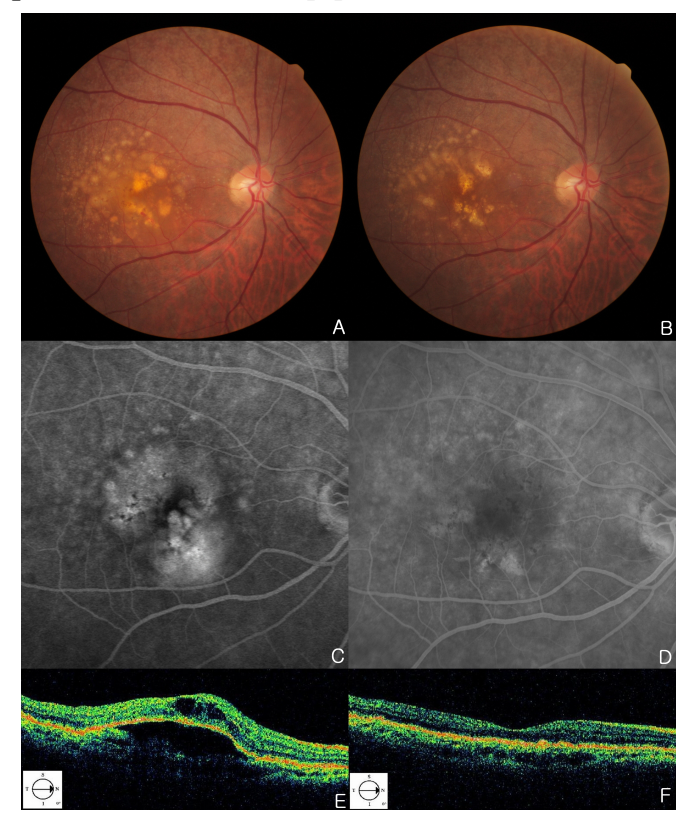

Fig. (1). (A, C, E) Fundus photographs (A), FFA (C) and OCT (E) images of patient at the first examination. Fundus photographs, a centrally located DPED are seen with diffuse coalesced soft drusen at right eye. Hyperfluorescence of FFA and hypo-reflectivity of OCT correspond to CNV with a DPED. (B, D, F) Fundus photographs (B), FFA (D) and OCT (F) images of patient after ranibizumab injections. After 5 intravitreal ranibizumab injections, six months later, DPED at foveal area and drusen located superotemporal to macula are diminished over time. The late-phase fluorescein study shows regression of CNV.

We could not completely rule out the possibility of a natural regression or dynamic changes of drusen and DPED. However, at the literature, natural course of drusen and DPED usually takes 2 to 5 years to change [1]. In our case, because diminishment of drusen and DPED took only 6 months, we have postulated anti-VEGF may have crucial role in these changes.

Mixture of multiple fundus findings was one more consideration in this case. In the case of a patient with a mixture of drusen, DPED and CNV, FAG and OCT may not be able to facilitate decisive diagnoses, as it is difficult to differentiate one from another with FAG and OCT. Thus, DPED with CNV looks larger than only DPED. In addition, decrease of DPED may be overestimated, after CNV regression.

The mechanism of influence of drusen and DPED after anti-VEGF therapy is uncertain. We have postulated a mechanism of reduction to explain our case reports. Possible mechanisms during and after intravitreal ranibizumab injection include mechanical and biochemical effects. Some stimulating factors have a common final pathway of reactive RPE changes resulting in phagocytosis of drusen material $[8,9]$. It may be speculated that nonspecific stimulating factors including mechanical (partial detachment of the posterior vitreous cortex, vitreoretinal traction), or biochemical (ranibizumab itself) factors. Also, the presence of lipid nature in the fluid component of pigment epithelial detachment may suggest a possible role for VEGF because VEGF is well known to control vascular permeability. We believe it is reasonable to postulate that the inhibition of VEGF by ranibizumab may decrease the influx of lipid and 
fluid by decreasing transvascular permeability [10]. Further, similar mechanisms may possibly lead to a decrease of lipid deposition from corneal neovascularization after anti-VEGF treatment [11, 12]. Gallego-Pinazo et al. [6] emphasized that the intravitreal ranibizumab seems to be beneficial in patients with DPED, especially when intraretinal fluid is present by OCT, like in our case. It cannot be confirmed whether DPED and drusen regression is good or poor prognostic factor to AMD. In our case, DPED and drusen were diminished. However, patients showed more severe findings of AMD such as CNV. Regression of DPED and drusen is induced in expectation that this will reduce the risk of CNV. Nevertheless, it shows that the regression of DPED and drusen should not be regarded as a recuperation from the disease. The correlation between drusen regression and CNV progression should be further studied.

In conclusion, intravitreal ranibizumab injection might be influenced dynamic changes of drusen and DPED. Further studies may need to provide insight into the pathogenesis of anti-VEGF in drusen and DPED.

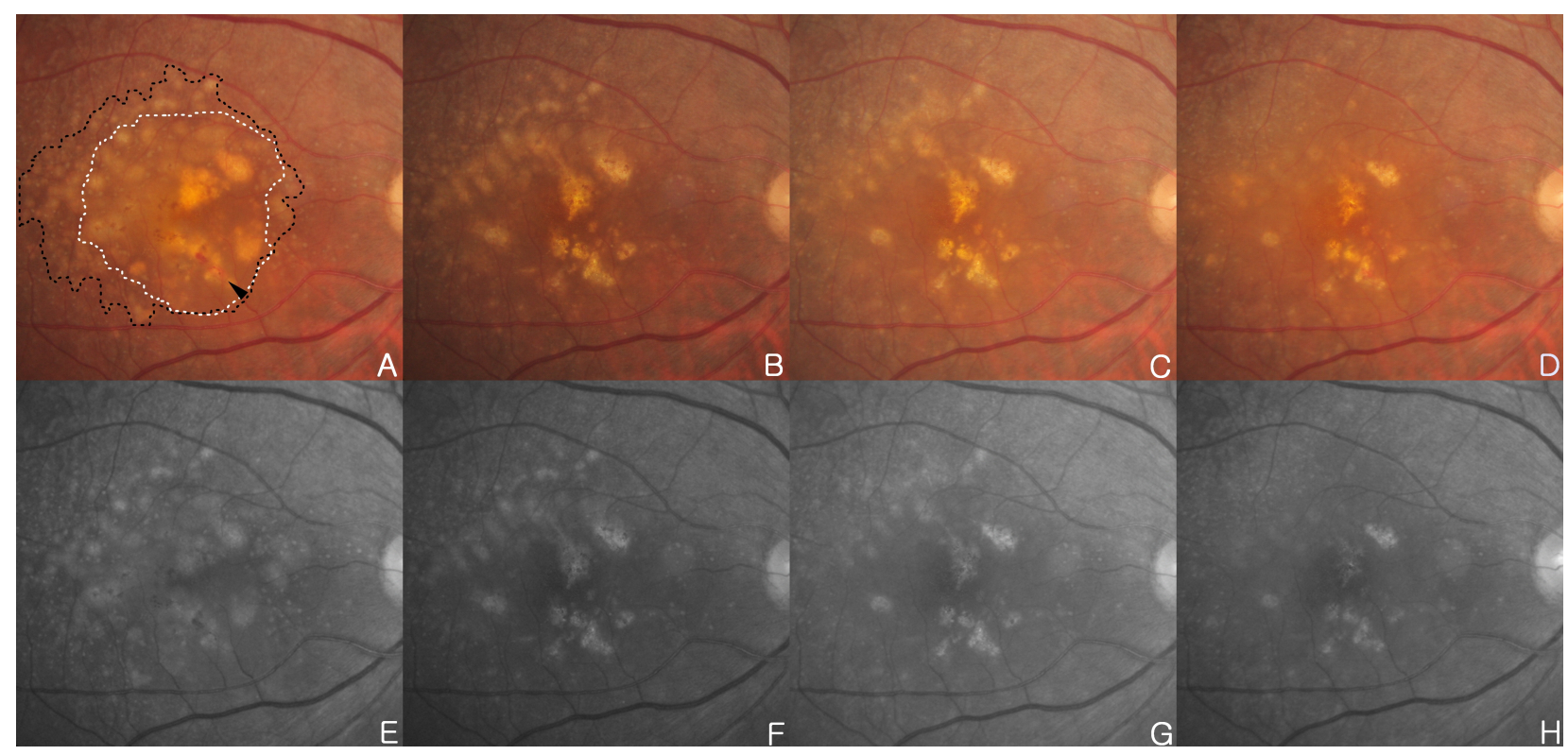

Fig. (2). (A, E) Initial examination, fundus photography and red free fundus image in the right eye revealed DPED (white dotted line) with diffuse coalesced soft drusen (black dotted line) at macular and paramacular region. There are focal hyperpigmentary changes and retinal hemorrhage (black arrowhead) over confluent drusen. (B, F) Six months after 5 intravitreal ranibizumab injections in the right eye, DPED at foveal area and drusens located superotemporal to macula are diminished over time. Retinal hemorrhage have also disappeared. (C, G) One month after the initial absorption of drusen and DPED, drusen and central DPED reduction continued. (D, H) Two months after initial absorption of drusen and DPED, previous central DPED reduction continued, and drusen at the superotemporal to macula area was decreased. But, CNV was recurred.

\section{CONFLICT OF INTEREST}

The authors confirm that this article content has no conflict of interest.

\section{ACKNOWLEDGEMENTS}

Declared none.

\section{REFERENCES}

[1] Cukras C, Agrón E, Klein ML, et al. Natural history of drusenoid pigment epithelial detachment in age-related macular degeneration: AgeRelated Eye Disease Study Report No. 28. Ophthalmology 2010; 117(3): 489-99.

[http://dx.doi.org/10.1016/j.ophtha.2009.12.002] [PMID: 20079925]

[2] Owens SL, Bunce C, Brannon AJ, et al. Prophylactic laser treatment hastens choroidal neovascularization in unilateral age-related maculopathy: final results of the drusen laser study. Am J Ophthalmol 2006; 141(2): 276-81. [http://dx.doi.org/10.1016/j.ajo.2005.08.019] [PMID: 16458680]

[3] Lee NY, Kim KS. Photodynamic therapy treatment for eyes with drusenoid pigment epithelium detachment. Korean J Ophthalmol 2008; 22(3): 194-6.

[http://dx.doi.org/10.3341/kjo.2008.22.3.194] [PMID: 18784450] 
[4] Rencová E, Bláha M, Studnička J, et al. Haemorheopheresis could block the progression of the dry form of age-related macular degeneration with soft drusen to the neovascular form. Acta Ophthalmol 2011; 89(5): 463-71. [http://dx.doi.org/10.1111/j.1755-3768.2009.01710.x] [PMID: 20102350]

[5] Krishnan R, Lochhead J. Regression of soft drusen and drusenoid pigment epithelial detachment following intravitreal anti-vascular endothelial growth factor therapy. Can J Ophthalmol 2010; 45(1): 83-4. [http://dx.doi.org/10.3129/i09-187] [PMID: 20130723]

[6] Gallego-Pinazo R, Marina A, Suelves Cogollos, et al. Intravitreal ranibizumab for symptomatic drusenoid pigment epithelial detachment without choroidal neovascularization in age-related macular degeneration. Clin Ophthalmol 2011; 5: 161-5. [PMID: 21383943]

[7] Querques G, Bux AV, Delle Noci N. Foveal geographic atrophy following intravitreal pegaptanib sodium (Macugen) for drusenoid pigment epithelium detachment. Eur J Ophthalmol 2009; 19(5): 890-3. [PMID: 19787618]

[8] Holz FG, Staudt S. Disappearance of soft drusen following macular hole surgery. Retina 2001; 21(2): 184-6 [http://dx.doi.org/10.1097/00006982-200104000-00018] [PMID: 11321151]

[9] Dithmar S, Pollithy S, Ach T. Disappearance of central confluent soft drusen following vitrectomy and ILM peeling. Eye (Lond) 2013; 27(6): $779-81$. [http://dx.doi.org/10.1038/eye.2013.35] [PMID: 23558209]

[10] Mathews MK, Merges C, McLeod DS, Lutty GA. Vascular endothelial growth factor and vascular permeability changes in human diabetic retinopathy. Invest Ophthalmol Vis Sci 1997; 38(13): 2729-41.

[PMID: 9418725]

[11] Chu HS, Hu FR, Yang CM, et al. Subconjunctival injection of bevacizumab in the treatment of corneal neovascularization associated with lipid deposition. Cornea 2011; 30(1): 60-6. [http://dx.doi.org/10.1097/ICO.0b013e3181e458c5] [PMID: 20847676]

[12] Yeung SN, Lichtinger A, Kim P, Amiran MD, Slomovic AR. Combined use of subconjunctival and intracorneal bevacizumab injection for corneal neovascularization. Cornea 2011; 30(10): 1110-4. [http://dx.doi.org/10.1097/ICO.0b013e31821379aa] [PMID: 21673570]

Received: July 5, 2015 Revised: October 30, 2015 Accepted: October 30, 2015

(C) Kim et al.; Licensee Bentham Open.

This is an open access article licensed under the terms of the Creative Commons Attribution-Non-Commercial 4.0 International Public License (CC BY-NC 4.0) (https://creativecommons.org/licenses/by-nc/4.0/legalcode), which permits unrestricted, non-commercial use, distribution and reproduction in any medium, provided the work is properly cited. 